OPEN ACCESS

\section{Extra physics with an ABS and a Lamb-shift polarimeter}

To cite this article: R Engels et al 2011 J. Phys.: Conf. Ser. 295012161

View the article online for updates and enhancements.

\section{Related content}

\author{
First experiments with the polarized \\ internal gas target at ANKE/COSY \\ M Mikirtychyants, $\mathrm{R}$ Engels, $\mathrm{K}$ Grigoryev et \\ Spin-filtering at COSY \\ Christian Weidemann and the PAX \\ collaboration \\ The polarised internal target for the PAX \\ G Ciullo, L Barion, C Barschel et al.
}

\section{Recent citations}

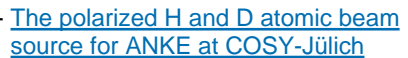
M. Mikirtychyants et al 


\title{
Extra physics with an ABS and a Lamb-shift polarimeter
}

\author{
R Engels ${ }^{1}$, R Emmerich Em Grigoryev $^{1,3}, \mathbf{P}$ Kravtsov $^{3}$, L Kochenda ${ }^{3}$, \\ M Mikirtytchiants ${ }^{1,3}, \mathbf{S}$ Paul $^{2}$, F Rathmann ${ }^{1}, \mathbf{H}$ Paetz gen. Schieck ${ }^{4}$, \\ W Schott ${ }^{3}$, G Schug ${ }^{1}$, H Seyfarth ${ }^{1}$, H Ströher ${ }^{1}$, V Trofimov $^{3}$, \\ A Vasilyev ${ }^{3}$, and M Westig ${ }^{5}$ \\ ${ }^{1}$ Institute for Nuclear Physics / JCHP, Forschungszentrum Jülich, 52425 Jülich, Germany \\ ${ }^{2}$ Physics Department E18, Tech. University Munich, James-Franck Str., 85748 Garching, \\ Germany \\ ${ }^{3}$ Petersburg Nuclear Physics Institute, Orlova roscha, 188300 Gatchina, Russia \\ ${ }^{4}$ Institute for Nuclear Physics, University of Cologne, Zülpicher Str. 77, 50937 Köln, Germany \\ ${ }^{5}$ I. Physics Institute, University of Cologne, Zülpicher Str. 77, 50937 Köln, Germany \\ E-mail: $r . w . e n g e l s @ f z-j u e l i c h . d e$
}

\begin{abstract}
The polarized internal gas target of the ANKE experiment is only used for a few months per year for hadron physics at the cooler synchrotron COSY. In the meantime, the whole setup or components like the ABS or the Lamb-shift polarimeter can be used for other experiments. We present various projects, from atomic and molecular physics to a neutrino experiment, for which the existing hardware can be used.
\end{abstract}

\section{Introduction}

During times when the polarized internal gas target (PIT) of the ANKE experiment, comprising a polarized atomic beam source (ABS) and a Lamb-shift polarimeter (LSP) [1], is not mounted inside the cooler synchrotron COSY, it is used for other purposes. For example, an ABS can be used to produce polarized deuteron(d) and Deuterium(D) beams to investigate the influence of the polarization on the total cross section of the dd-fusion reactions at low energies where thermonnuclear fusion reactors will work in the future [2]. Below, we present other projects, which we are going to pursue using the existing or equivalent hardware.

\section{Hydrogen spectroscopy}

With the spinfilter [3], the central component of the Lamb-shift polarimeter [4], it is possible to produce a beam of metastable hydrogen (deuterium) atoms in just one Zeeman state. With induced single transitions between the different Zeeman states of the $2 S_{1 / 2}$ and the $2 P_{1 / 2}$ hyperfine states, the Breit-Rabi diagrams, including the hyperfine energy splittings, the Lamb shift and the Lande factors can be measured very precisely.

\subsection{The Breit-Rabi diagram of the $2 S$ state of hydrogen and deuterium}

With a setup similar to that of the Atomic Beam Resonance Method [5] (see Fig. 1), the complete Breit-Rabi diagram of the $2 S$ state of hydrogen and deuterium can be measured. In our setup, 


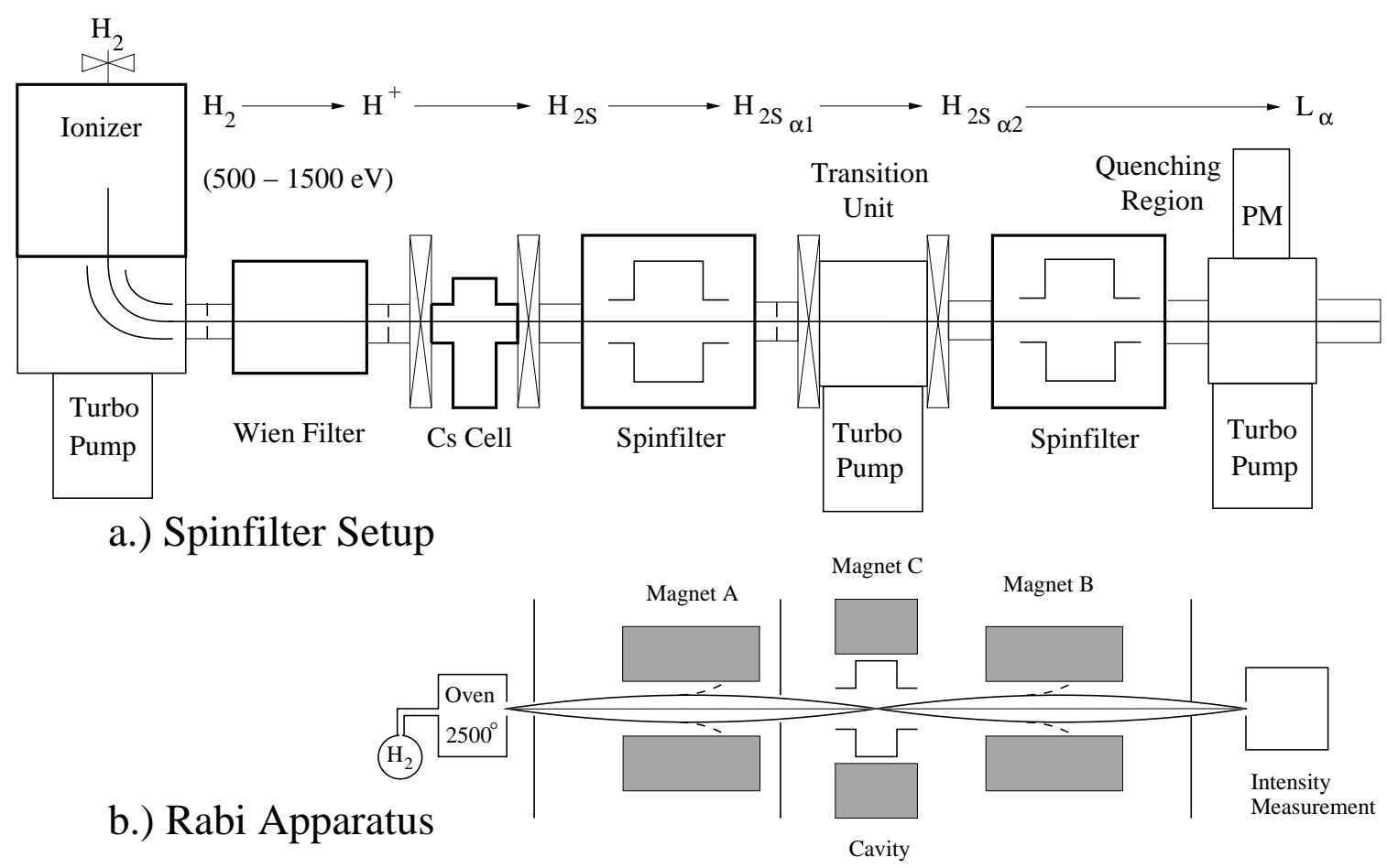

Figure 1. a) The setup for the measurement of the complete Breit-Rabi diagram of the $2 S$ states of hydrogen or deuterium atoms. b) The schematic view of the classical Rabi apparatus.

the analyzing magnets of the Rabi apparatus are replaced by spinfilters.

In an electron-impact ionizer $\mathrm{H}_{2}\left(\mathrm{D}_{2}\right)$ molecules are dissociated and ionized. With acceleration of the ions to energies between 300 and $2000 \mathrm{eV}$, beam intensities up to $10 \mu \mathrm{A}$ can be achieved. After deflection to a horizontal beam direction, a Wien filter is used to separate the protons from the other ions, which originate from the residual gas. By charge exchange with cesium vapour [6], metastable hydrogen atoms in the state $2 S_{1 / 2}$ are produced from about $15 \%$ of the protons. In the first spinfilter all metastable atoms except those in one Zeeman state are quenched into the ground state. Only metastable atoms in the Zeeman states $\alpha 1, \alpha 2$ or, using a subsequent Sona transition, $\beta 3$ remain in the beam. In a homogeneous magnetic field, magnetic dipole transitions are induced with the magnetic field vector parallel to the velocity direction of the hydrogen atoms in order to avoid longitudinal Doppler shift and broadening. The direction of a static magnetic field, produced by two Helmholtz coils, can be aligned either parallel or perpendicular to the velocity direction of the atoms. Thus, for a transverse magnetic field, the transitions $\alpha 1 \rightarrow \alpha 2, \alpha 1 \rightarrow \beta 4, \alpha 2 \rightarrow \alpha 1$ and $\alpha 2 \rightarrow \beta 3$ can be measured. The second spinfilter is used to verify the induced transitions. As an example in Fig. 1 atoms in the metastable state $\alpha 1$ are leaving the first spinfilter and transfered into $\alpha 2$ in the transition unit. The second spinfilter is set to transmit the state $\alpha 2$ only. Therefore, metastable atoms can produce light in the quenching chamber only if the transition from state $\alpha 1$ into state $\alpha 2$ has occured in the transition unit. The large number of at least $10^{5}$ photons $/ \mathrm{s}$ detected in the photomultiplier allows to reach a statistical error comparable to that of the best measurements [7] in $20 \mathrm{~min}$. In addition, the hyperfine-splitting energy can be measured independently of the magnetic field as a combination of the transition frequencies $(\alpha 1 \rightarrow \beta 4)-(\alpha 2 \rightarrow \beta 3)$ or $(\alpha 1 \rightarrow \alpha 2)+(\beta 3 \rightarrow \beta 4)$. Therefore, a huge number of individual and direct measurements is possible. To increase the precision of the experiment the halfwidth of the measured resonances can be decreased with use 


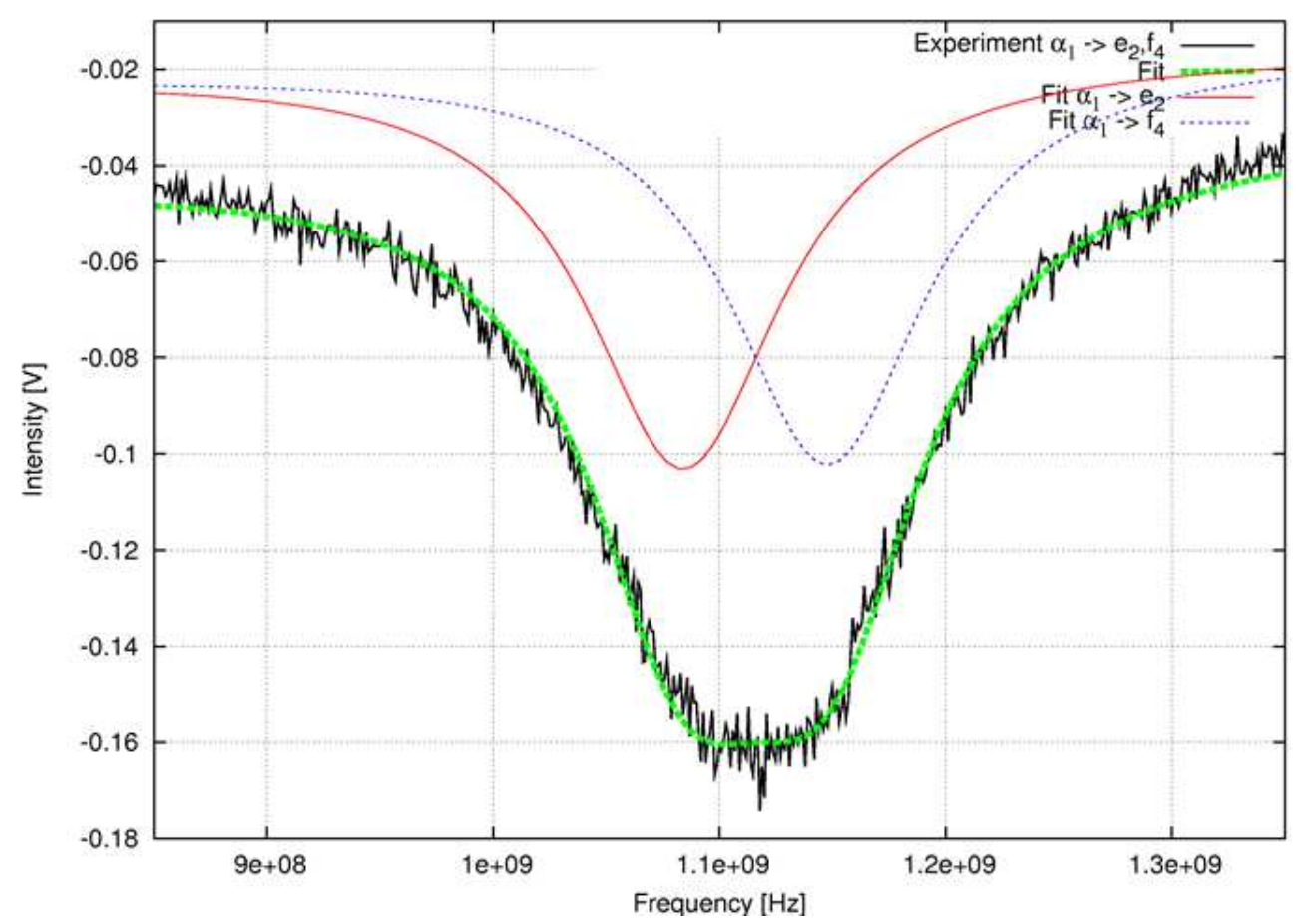

Figure 2. The observed transitions $(\alpha 1 \rightarrow f 4)$ (blue) and $(\alpha 1 \rightarrow e 2)$ (red) at a small vertical magnetic field in the transition region. The green function is a fit on the measured data (black) from which the resonance signals of both transitions can be calculated separately.

of the so called separated oscillatory field method [8] and with much slower metastable hydrogen beams produced, e.g., with the ABS and excited by electron bombardment. By that, it will be possible to reach the precision of current laser-spectroscopy experiments and to measure the g-factor of the $2 S$ state with a precision of $10^{-8}$.

\subsection{The Breit-Rabi diagram of the 2P state of hydrogen and deuterium}

With induced electric-dipole transitions from the single $2 S$-Zeeman states into single $2 \mathrm{P}$ states behind the spinfilter, the classical Lamb shift [9] and the complete Breit-Rabi diagram of the $2 \mathrm{P}$ state can be measured. In contrast to the original Lamb measurements today it is possible to change the RF frequency without changing of the RF power in a constant magnetic field by a Lecher TEM waveguide.

In a proof-of-principle exoeriment [10] metastable hydrogen atoms in the HFS $\alpha 1$ or $\alpha 2$ are selected in the spinfilter and reach the TEM waveguide. For a small transverse magnetic field close to $0 \mathrm{G}$ the transitions $(\alpha 1 \rightarrow f 4)$ and $(\alpha 1 \rightarrow e 2)$ (Fig. 3, left) or $(\alpha 2 \rightarrow f 3)$ and $(\alpha 2 \rightarrow e 1)$ (Fig. 3, right) are observed. The difference between the two resonances of the transitions $(\alpha 1 \rightarrow f 4)$ and $(\alpha 1 \rightarrow e 2)$ corresponds to the hyperfine splitting energy of the $2 P_{1 / 2}$ state. The result from a fit of $60(2) \mathrm{MHz}$ agrees with the earlier result of 59.22 (14) $\mathrm{MHz}[8]$. The transition frequencies from the $2 S_{1 / 2}$ into the $2 P_{1 / 2}$ state together with the HFS of these states allows to obtain the classical Lamb shift. As a first result a value of $1057(1) \mathrm{MHz}$ could be determined. The dominant error, which is up to 3 orders of magnitude larger compared to the best values, is produced by the inhomogeneity and the uncertainty of the magnetic field. An error of $\Delta B=0.5 \mathrm{G}$, e.g., corresponds to an error of $\Delta f=1 \mathrm{MHz}$. 


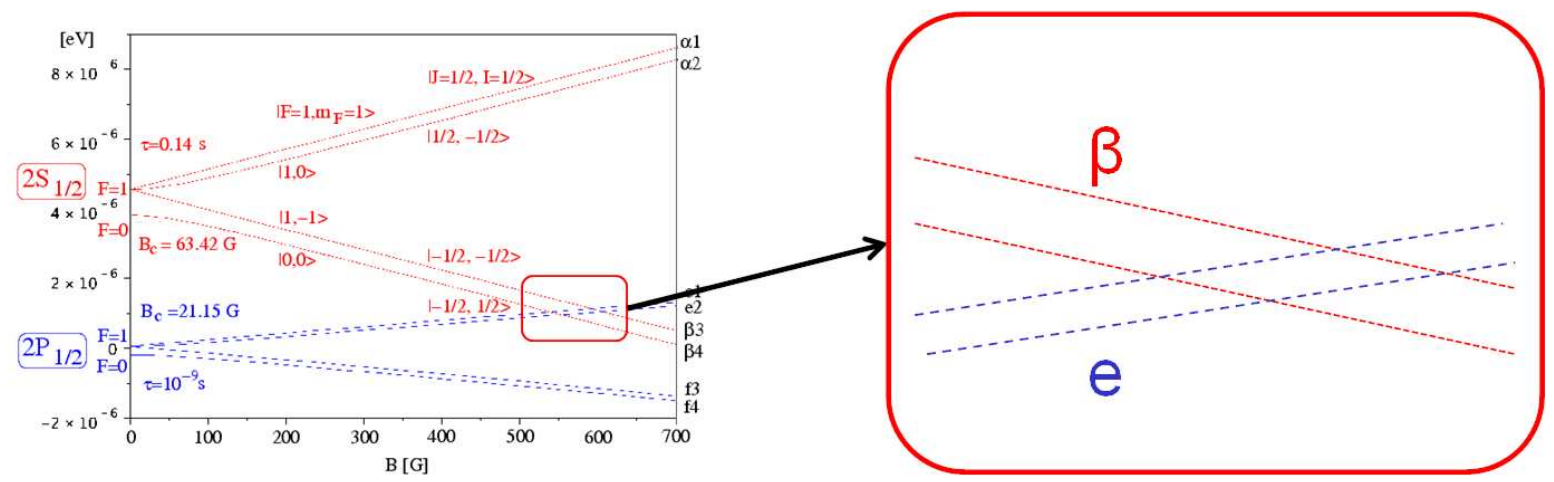

Figure 3. The crossing points of the $2 S_{1 / 2}$ and the $2 P_{1 / 2}$ hyperfine substates around $57 \mathrm{mT}$.

\subsection{Parity violation in the hydrogen atom}

At the crossing points of the $\beta$-substates of the $2 S_{1 / 2}$ state and the $e$-substates of the $2 P_{1 / 2}$ around $57 \mathrm{mT}$ (see Fig. 3) and of the $\beta$-substates and the $f$-substates at $120 \mathrm{mT}$ in the BreitRabi diagram direct transitions between the states are forbidden by parity conservation of the electromagnetic interaction. However, the electroweak interaction of the standard model leads to a small admixture of the parity-violating weak force [11]. From this, all four electron-nucleon and weak-neutral-current coupling constants $C_{1 p}, C_{2 p}, C_{1 n}$ and $C_{2 n}$ can be deduced, if such experiments are made for hydrogen and deuterium in parallel, when $C_{1 D}=C_{1 p}+C_{1 n}$ and $C_{2 D}=C_{2 p}+C_{2 n}$ are defined for deuterium.

With the equation: $2 C_{1 p}=Q_{W}(p)=\left(1-4 \sin ^{2} \Omega_{W}\right)$ the weak charge of the proton $Q_{W}(p)$ and the weak mixing angle $\sin ^{2} \Omega_{W}$ are measured for very low momentum transfer and, therefore, the standard model can be tested.

The methods, used until now to observe these transitions, can be copied and optimized with the components of a Lamb-shift polarimeter to increase the intensity by several orders of magnitude. But with a spinfilter a more direct technique is possible: The ionizer in combination with the Wien filter, the Cs cell and a Sona transition will be used to produce an intense beam of metastable hydrogen atoms in the states $\alpha 1, \alpha 2$ or $\beta 3$. For deuterium the states $\alpha 1, \alpha 2, \alpha 3$ or $\beta 4$ are possible. The missing states $\beta 4$ for hydrogen and $\beta 5$ and $\beta 6$ for deuterium can be induced like the M1 transitions in an ABS. These atoms will be transmitted through a magnetic field, which can be continuously changed between 50 and $65 \mathrm{mT}$ or around $120 \mathrm{mT}$. Inside the magnetic field, will be an ellipsoidally-shaped body made from aluminum for two different reasons. In the inner part of this body is no electric field to allow transitions from the $2 S_{1 / 2}$ states into the $2 P_{1 / 2}$ states by Stark-effect. The inner surface will reflect Lyman- $\alpha$ photons until they are collected by a photomultiplier outside the magnetic field. With this mirror around the interaction region the efficiency for the detection of the Lyman- $\alpha$ photons will be increased to $5 \%$. Now, the occupation numbers of the different hyperfine substates in front of this interaction region are changed by the spinfilter, the Sona or the $M 1$ transition and the magnetic field is continuously changed through the crossing points around 54 or $120 \mathrm{mT}$. With the help of the substates $\alpha 1$ and $\alpha 2$ the background in the multiplier signal can be monitored and when the states $\beta 3$ or $\beta 4$ are used a signal at $54 \mathrm{mT}$ or $120 \mathrm{mT}$ might appear for hydrogen. The Sona transition unit will allow transitions from state $\alpha 1$ into $\beta 3$, but no transitions from $\alpha 2$ into a $\beta$-state. Therefore, the influence of switching on and off of the Sona transition is visible, too. In addition, a huge number of data sets can be averaged and with the use of a lock-in amplifier the sensitivity of the experiment will increase. With $10^{13}$ metastable atoms/s in one single hyperfine state in the interaction region in one week $10^{18}$ metastable atoms have passed the 


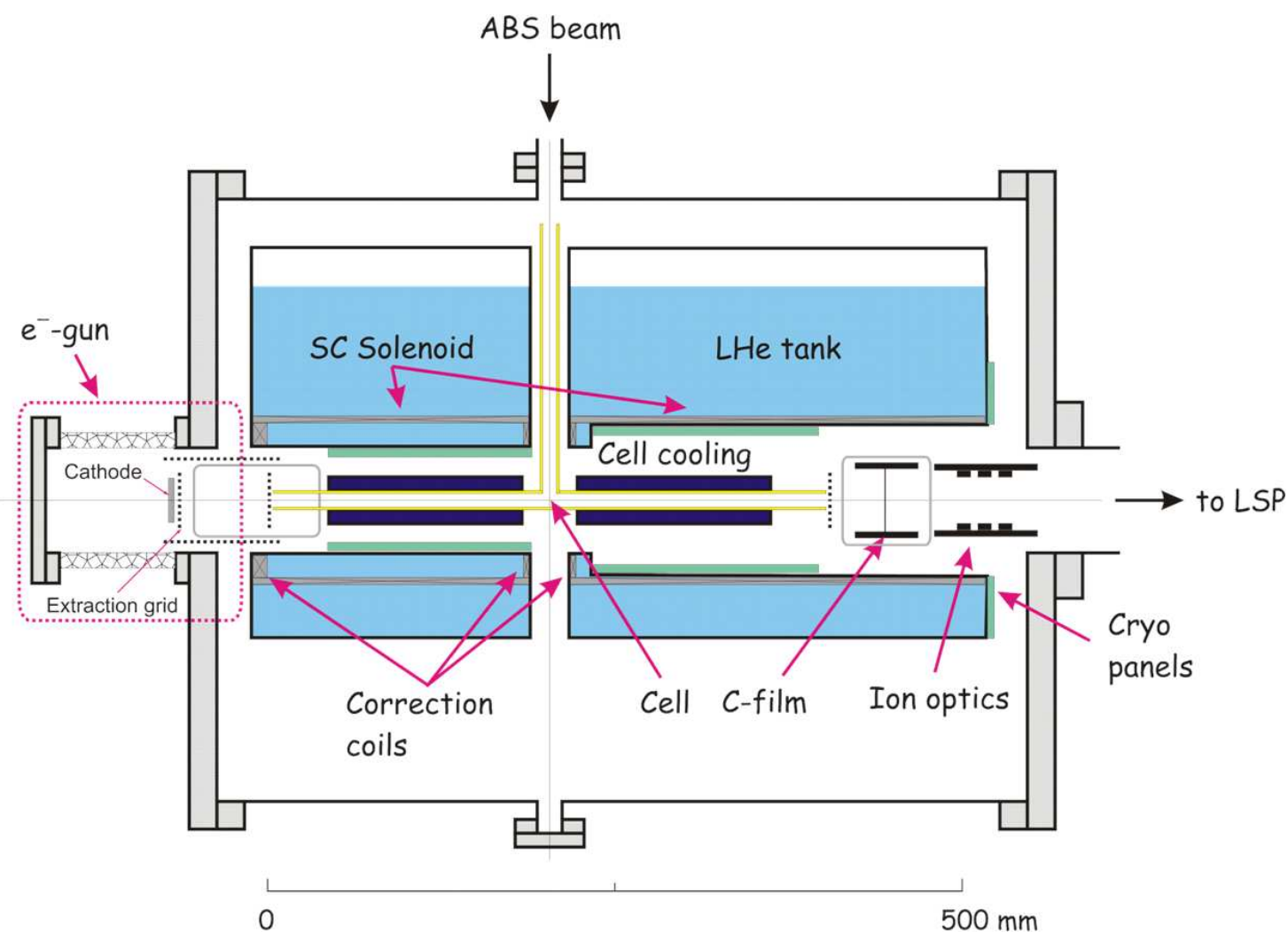

Figure 4. Setup of the experiment to measure the polarization of hydrogen (deuterium) molecules after recombination of polarized atoms.

photomultiplier in one out of the 4 different combinations of spinfilter resonances $(\alpha 1$ and $\alpha 2)$ and Sona transition on or off (on: $\beta 3$ or $\alpha 2 /$ off: $\alpha 1$ or $\alpha 2$ ) or induced $M 1$ transition. Due to the quantum efficiency of the photomultiplier $(10 \%)$ one out of 10 photons in the photocathode will be registered. One order of magnitude is lost because some photons will leave the interaction region while they are not reflected to the photomultiplier and by fluctuation of the background signals. Here, it should be mentioned that a photomultiplier which exists of a photocathode of $\mathrm{KBr}$ and an entrance window made from $\mathrm{MgF}$ is optimized for Lyman- $\alpha$ photons $(121 \mathrm{~nm}$ $\leftrightarrow 10 \mathrm{eV}$ ) and has no dark current even without cooling and a tiny sensitive range between 115 and $135 \mathrm{~nm}$. Thus, a relative uncertainty for the appearance of these transitions of $10^{-15}$ or better seems possible within one week of measurements. The major source for background signals at the photomultiplier will be scattering of beam atoms with residual gas inside the aluminum ellipsoid. Therefore, a vacuum pressure below $10^{-8}$ mbar or better is essential. With this improvements an uncertainty smaller than the values of the parity mixing matrix elements and, therefore, of the weak mixing angle $\sin ^{2} \Omega_{W}$ is possible.

\section{Polarized molecules}

When polarized hydrogen atoms recombine in a storage cell, the residual $H_{2}$ molecules may still be nuclear polarized [12]. In a collaboration between PNPI, Gatchina, University of Cologne and FZ Jülich a device was built (see Fig. 4) to measure the polarization of hydrogen atoms and hydrogen molecules after recombination of polarized atoms depending on different materials, temperatures and magnetic fields. In a superconducting solenoid polarized atoms from the ABS 


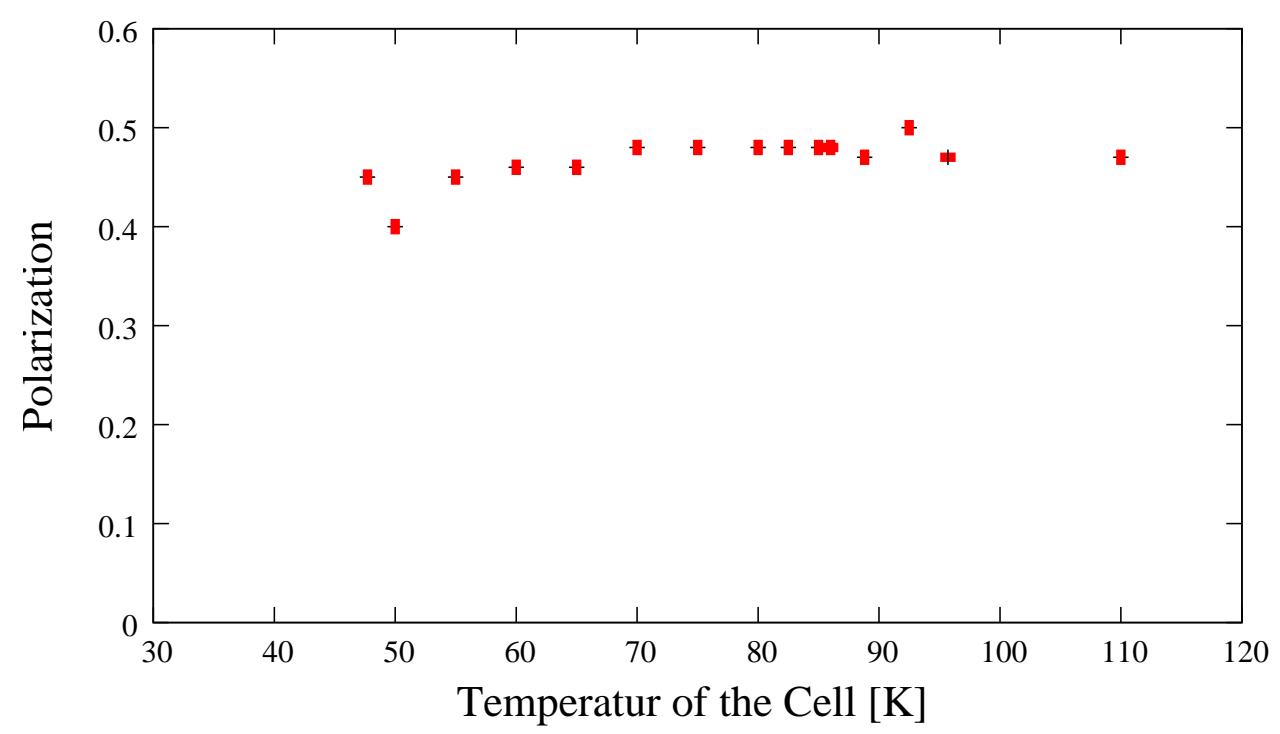

Figure 5. One of the first results with polarized hydrogen atoms in a storage cell with a gold surface. The polarization for atoms in HFS 1 is stable and independent of the temperature. (Magnetic field: $0.28 \mathrm{~T}$, ion beam energy: $4 \mathrm{keV}$ )

partly recombine in a T-shape storage cell, where the inner surface can be covered with different materials. Both, atoms and molecules, are ionized afterwards by electron bombardment and the protons and $\mathrm{H}_{2}^{+}$ions produced are accelerated to an energy of a few keV. Inside the solenoid both ions have to pass a thin carbon foil, where the last electron of the $\mathrm{H}_{2}^{+}$ions is stripped off and, therefore, two protons are produced. These protons share the kinetic energy of the $\mathrm{H}_{2}^{+}$ ion and can be separated by the Wien filter of the LSP from the protons, which originate from the initial atoms. In this way, the nuclear polarization of the atoms and the molecules can be measured under various conditions.

After solving a long list of minor problems, in the summer of 2009 first experiments were performed on a gold surface. They showed the surprising result shown in Fig. 5. The polarization for a fixed magnetic field was stable even at low temperatures as low as $47 \mathrm{~K}$. Until now, no material has been found which could preserve the polarization for hydrogen atoms at temperatures below $60 \mathrm{~K}[12]$.

\section{Rare neutron decay}

In the neutron decay $n \rightarrow p+e+\bar{\nu}_{e}$ the proton and electron can be found in different bound $\mathrm{S}$ states of the hydrogen atom [13]. The kinetic energy of $326.5 \mathrm{eV}$ for this 2-body decay fits to the energy range of the Lamb-shift polarimeter. Therefore, the polarization of the metastable hydrogen atoms can be measured, i.e., the proton and electron spin after the decay. If the helicity of the antineutrino is completely positive (right-handed), then the probabilities to find the different combinations of electron and proton spin, i.e. the different Zeeman states of the hydrogen atom, can be calculated and tested with this experiment. For example, the state $\beta 3$ is not allowed for the outcoming metastable atoms. Therefore, left-handed admixtures to the helicity or scalar and tensor contributions to the weak force can be measured with high precision $[14,15]$. The challenge of the experiment is the low count rate of metastable hydrogen atoms behind the spinfilter. For the through-going FRM II beam tube less than 1 count per 10 second is expected. For the detection of the outgoing atoms 4 different methods are suggested: 
- Ionization of the metastable atoms only by 2 different lasers and collection of the outcoming protons (Efficiency: $\sim 50 \%$ ).

- Detection of the Lyman- $\alpha$ photons after quenching the metastable atoms with a photomultiplier and a setup of optimized mirrors (Efficiency: $\sim 5 \%$ ).

- Detection of the Lyman- $\alpha$ photons by photoeffect on a CsI or a tungsten surface and collection of the electrons with a channeltron (Efficiency: > 50\%).

Meanwhile, it could be shown that the electron production efficiency of the Lyman- $\alpha$ photons on a tungsten surface is in the order of $10^{-3}$, but on a $C s J$ surface an efficienciy of $50 \%$ could be reached.

- Selective charge exchange of metastable hydrogen atoms with argon and separation of the $H^{-}$ions produced by a velocity filter (Efficiency: $\sim 10 \%$ ).

In a proof-of-principle experiment it was shown, that the kinetic energy of the $H^{-}$ions after the charge exchange depends on wether the atoms infront of the Argon cell are exited or not. The expected energy difference of $10.1 \mathrm{eV}$ was measured and a paper is in preparation.

Which method will give the best signal-to-noise ratio and a reasonable efficiency will be tested at the Technical University of Munich, Physik Department E18 and the Max Planck Institute for Quantum Optics in Munich.

\section{References}

[1] M Mikirtytchiants et al. 2011 contribution to this proceedings

[2] K Grigoryev et al. 2011 contribution to this proceedings

[3] J L McKibben et al. 1969 Phys. Lett. 28B 594

[4] R Engels et al. 2003 Rev. Sci. Instr. 74114607

[5] I I Rabi et al. 1939 Phys. Rev. 55526

[6] P Pradel et al. 1974 Phys. Rev. A 10797

[7] N Kolachevsky et al. 2009 Phys. Rev. Lett. 102213002

[8] S R Lundeen et al. 1975 Phys. Rev. Lett. 34377

[9] W E Lamb and R C Retherford 1951 Phys. Rev. 81222

[10] M Westig et al. 2010 Eur. Phys. J. D 5727

[11] R W Dunford and R J Holt 2007 J. Phys. G 342099

[12] T Wise et al. 2001 Phys. Rev. Lett. 87042701

[13] L L Nemenov 1980 Sov. J. Nucl. Phys. 31115

[14] W Schott et al. 2009 Hyperfine Int. 193, 269

[15] W Schott et al. 2006 Eur. Phys. J. A 30603 